\title{
FROM SWITCHING ARCS TO BALL LIGHTNING TO CURING CANCER
}

\author{
J. J. LOWKE
}

CSIRO Manufacturing, PO Box 218, Lindfield NSW 2070, Sydney, Australia

John. Lowke@csiro. au

\begin{abstract}
Previous modelling of switching has been through calculation of reductions in temperature of the arc at "current zero". "Enthalpy density"as a function of temperature is found to be an important property. New calculations now include an account of non-equilibrium electron density as a function of time through current zero and it is found that electron attachment rates, which are very large for $\mathrm{SF}_{6}$, could be a dominant property. Modelling discharges is having other successes, for example in explaining "ball lightning" observations inside of houses and aircraft, which suddenly appear, usually at glass windows. Discharge modelling suggests these observations might be explained by the production of "singlet delta"metastable molecules of oxygen in electrical discharges in air. If metastable densities are sufficient, electrons can be produced from the detachment of negative ions to produce radiation and explain ball lightning. An exciting new development is that plasmas from electric corona in air have been found to reduce the size of cancer tumours. These excited oxygen molecules have also been proposed as having a role in this remarkable interchange between classical electrical engineering and medical science.
\end{abstract}

Keywords: switching arcs, current zero, $\mathrm{SF}_{6}$, ball lightning, cancer.

\section{Introduction - Computer Modelling}

Naturally, of principal importance in the world, is "hardware", be it from the buildings of cities, power stations, the circuit breakers that control the power stations, or the iPhones that we all now use for everyday communications. But there is a new activity, only developed in the last 60 years of our civilisation, and that is computer modelling. There are hundreds of thousands, of people now involved in computer modelling which is also a principal activity behind a major fraction of the papers of this conference. Computer modelling has resulted in predictions of climate change, which are influencing economic policies of a significant fraction of the world. Computer modelling, based on previous representations of the world through mathematics, for example in the equations of electro magnetism, have now resulted in predictions of the very complex phenomenon of circuit interruption. Through the development of computers, predictions of the original analytic formula from mathematics have been extended to include numerical results giving details, frequently in three dimensions, of arc behaviour, be it interrupted or not, as a function of time.

Such predictions for switching arcs, based on the modelling of arc temperature, are discussed in Section 2. A new exciting development of such predictions of arc switching, is the inclusion of predictions of the electron density, discussed in Section 3. A further product of computer modelling is presented in Section 4, namely a proposed explanation of the centuries old phenomenon of Ball Lightning. This explanation results from the inclusion in the modelling of predictions of an additional particle to the particles that are usually considered, namely electrons, posi- tive ions and negative ions. This additional particle is the metastable excited state of oxygen molecules, which have the technical name 'singlet delta'oxygen molecules. Section 2, relates modelling achievements of the past, whereas Sections 3 and 4 describe present developments of computer modelling. Section 5 outlines an area, which I am sure will result in future computer modelling, namely the use of gas discharges for the curing of cancer! It has recently been found that electrical discharge plasmas, when in contact with cancer tumours, result in a reduction in the size of the tumours, including also the possible reduction of the size of secondary tumours. Such a phenomenon raises the possibility of a completely new field: the interaction of electrical engineering plasmas with biological systems, for example through the interaction with blood and human systems of circulation.

\section{Switching predictions from arc temperature}

In a switch, or "circuit breaker", two electrodes are separated to produce an arc, which ideally is extinguished at the next current zero of the ac cycle because of the cooling of the arc during the low electric field that occurs at current zero. With increases in population and increased demands for electric power from power stations there have been increased demands on the capacity of circuit breakers. As a result there have been continuous improvements with time in, for example, the design of electrode configurations, the arc gas composition and details of imposed convective flow, all largely achieved by experiment.

With the development of computers in the 1960's, there were efforts to "model"the whole circuit inter- 


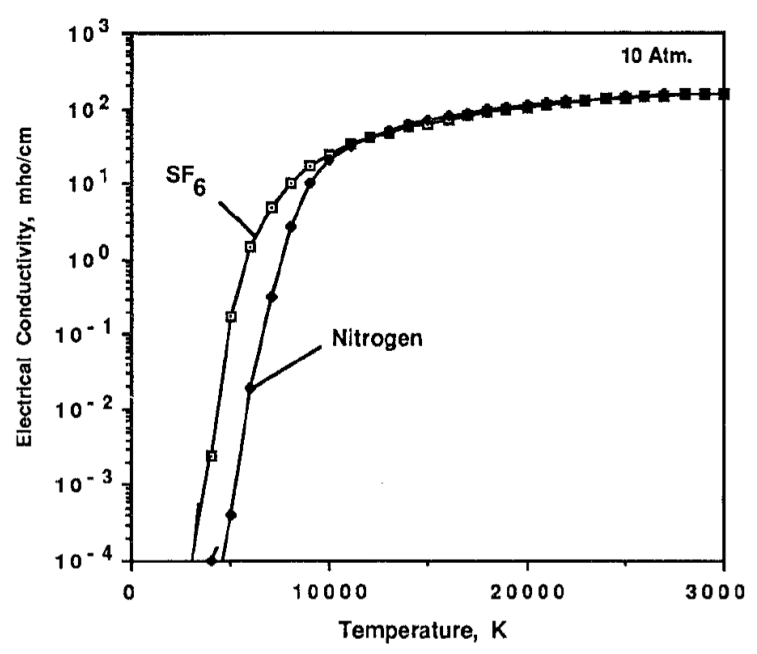

Figure 1. Equilibrium electrical conductivity; 1 bar.

ruption process with computers. Details of arc cooling by the processes of conduction, convection and radiation could all be modelled on a computer, as material functions of arc properties such as thermal and electrical conductivities and radiation emission coefficients were all known or could be calculated as a function of temperature and pressure. Then two-dimensional predictions of arc temperatures were made as a function of time to predict whether arc extinction would be achieved.

Figure 1 shows calculated equilibrium electrical conductivities for the two common circuit breaker gases of air and $\mathrm{SF}_{6}$ as a function of pressure at 1 bar. It is seen that if cooling to temperatures below $1000 \mathrm{~K}$ is achieved the electrical conductivity is near zero and circuit interruption is achieved and current is not possible. Thus it was envisaged that proposed modifications in design could be modelled first theoretically on the computer and the viability of the modifications could be tested much more cheaply than by an experimental trial.

Results of such modelling based on predictions of arc temperature have been very successful in predicting arc diameters for approximately steady state arcs at high current and have been used particularly for the design of "puffer" circuit breakers, where the ablation caused by high currents could be predicted by predictions of arc diameters. These 'temperature'models are also successful in making predictions of circuit interruption, which is a significant achievement. However, early predictions shown in Fig. 2 for conditions of imposed high speed flow showed large differences between theory and experiment and also nitrogen as a better interruption gas than $\mathrm{SF}_{6}$ ! Subsequent workers have removed the disparity of such theoretical predictions by introducing turbulence losses into the energy balance equation. But for results to give the much higher interruption ability for $\mathrm{SF}_{6}$ compared with air or nitrogen, the turbulence parameters for $\mathrm{SF}_{6}$ need to be significantly higher than for nitrogen or air, with

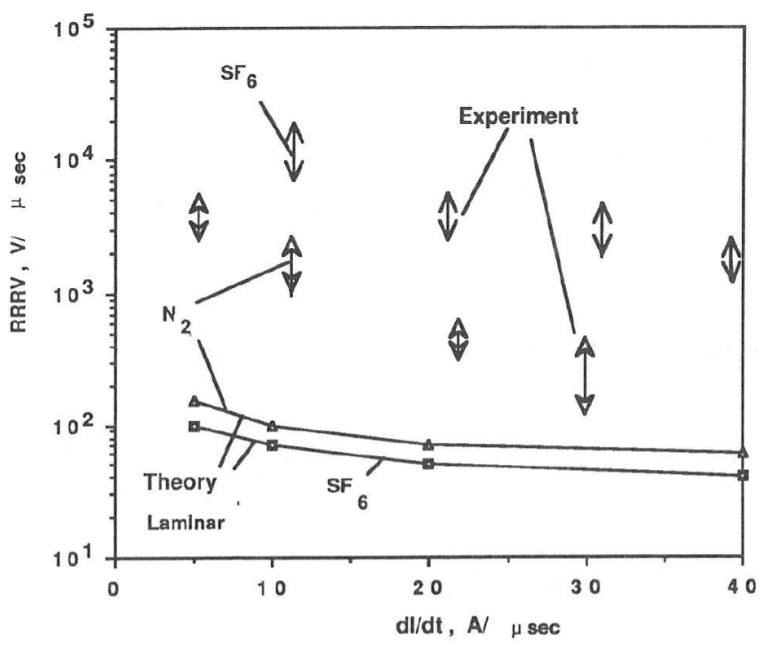

Figure 2. Calculations of circuit interruption for laminar flow [1].

no clear knowledge as to the physical reason for such a difference. One of the reasons for theory predicting superior interruption for nitrogen in Fig. 2 follows just from Fig. 1, where electrical conductivities for nitrogen are lower than for $\mathrm{SF}_{6}$ at low temperatures. There are further difficulties in predicting the pressure dependence of interruption when use is just made of the energy balance equation for arc temperature. The calculations assume that the electron density is just determined by the equilibrium value for the arc temperature.

\section{Switching arc predictions including predictions of electron densities}

In this section new calculations are presented which also include a solution for the time dependence of the electron density to obtain the non-equilibrium electrical conductivity. Predictions of electrical current and its interruption depend more directly on the electron density than the arc temperature. The electron density is determined by the balance between electrons produced by ionization and losses of electrons by electron attachment to neutral molecules and also recombination of electrons and positive ions. Thus three additional completely different material properties are involved in the theoretical predictions, namely the coefficients of ionization, attachment and recombination. Electron ionization and attachment coefficients are shown in Fig. 3 and it is seen that attachment for $\mathrm{SF}_{6}$ is generally more than 100 times larger for $\mathrm{SF}_{6}$ than for air. Furthermore the critical electric field for which ionization is larger than attachment is about four times larger for $\mathrm{SF}_{6}$.

A remarkable solution of the approximate relationship defining electron recombination as a function of time, namely $\mathrm{d} n_{e} / \mathrm{d} t=-\gamma n_{e} n_{+}=-\gamma n_{e}^{2}$ is that for a cooling plasma after time $t$, the electron density $n_{e}=1 / \gamma t$, regardless of the initial electron density. 


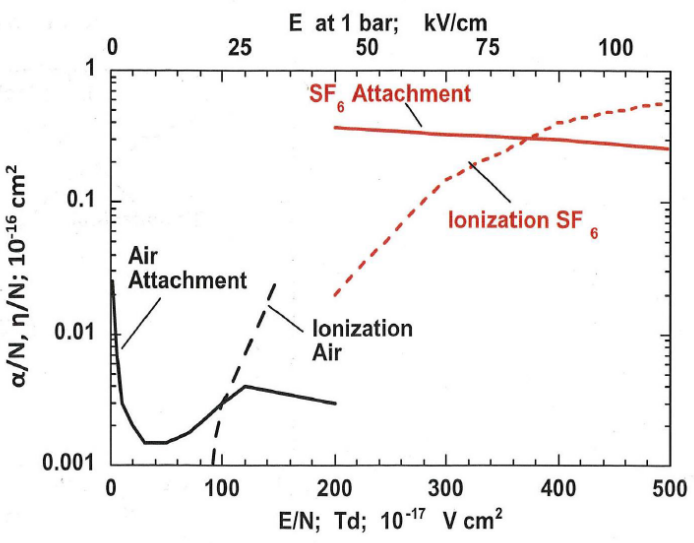

Figure 3. Ionization and attachment coefficients; air and $S F_{6}$.

This formula using a decay time of $1 \mu$ s and the large recombination coefficient, $\gamma$, of $10^{-7} \mathrm{~cm}^{3} . \mathrm{s}^{-1}$ gives an electron density of $10^{13} \mathrm{~cm}^{-3}$, nowhere near the zero electron density obtained from equilibrium plasmas of low temperatures. Thus circuit interruption will be determined by whether electron attachment can convert the excess non-equilibrium electrons that remain at current zero to negative ions. This physical picture is completely different from the physical picture obtained from the calculation of arc temperatures, which is a "heat transfer"model, depending on whether there is sufficient cooling to extinguish the arc.

Predictions of circuit interruption are then quite different, as shown in Fig. 4. The predicted critical rate of rise of recovery voltage (RRRV) for $\mathrm{SF}_{6}$ is $50 \mathrm{kV} . \mathrm{s}^{-1}$ compared with $8 \mathrm{kV} . \mathrm{s}^{-1}$ for air largely because of the higher attachment rate for $\mathrm{SF}_{6}$. When the pressure of $\mathrm{SF}_{6}$ is increased to 10 bar from 1 bar the RRRV increases to $200 \mathrm{kV} . \mathrm{s}^{-1}$, again largely because increased attachment occurs at the higher pressure. The predictions of Figure 4 apply for a low current arc, similar to that used in welding, with very little imposed flow, and clearly predict superior interruption for $\mathrm{SF}_{6}$ and increased pressure. Large circuit breakers used in power stations involve imposed gas flow at super-sonic velocities, in which case the loss term from the flow of the enthalpy of the arc will be very much larger and results may be very different from those of Fig. 4.

\section{Explanation of Ball Lightning from $\mathrm{a}^{1} \Delta_{\mathrm{g}}$ oxygen}

Ball lightning is occasionally seen during thunderstorms as a ball about the size of an orange, emitting light that can be easily observed, but without damage to the eyes. The balls move at about walking speed, usually about a metre above the ground and last about 10 seconds. Remarkably they have been observed most often inside of houses and even aeroplanes, frequently appearing from closed glass windows, usually without

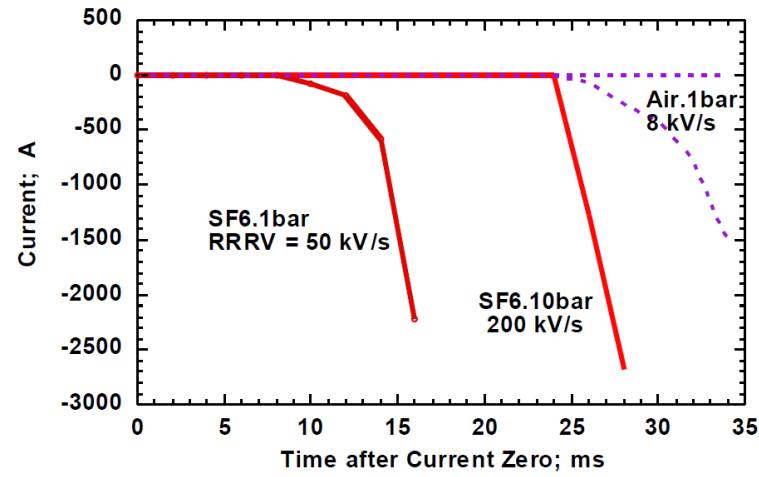

Figure 4. Calculated rates of rise of recovery voltage; from [2].

any remaining trace. Theories abound for the energy source, usually from something burning, such as silicon vapour, but also from microwaves, nuclear energy, dark matter, antimatter, masers and runaway electrons from outer space. In this author's view, none of these theories give a viable mechanism for producing such an energy source inside of houses or aeroplanes, almost always leaving no trace, such as an oxidation residue. The present proposal, though unlikely, does give such a model for ball-lightning, consistent with existing established concepts of electrical gas discharge behaviour.

The numerical predictions of the previous section 3 , involve predictions of the behaviour of electrons, positive ions and negative ions. The new analysis involves the inclusion of an additional particle, the metastable state of oxygen, referred to as "singlet delta" oxygen molecules, represented by $\mathrm{a}^{1} \Delta_{\mathrm{g}}$. This molecule has well established properties, namely an energy of almost exactly $1 \mathrm{eV}$, a radiative lifetime of about an hour, a lifetime at atmospheric pressure of seconds, known cross sections as a function of energy for excitation by electrons, and also known quenching rates in air and also by water vapour. Of great significance, this metastable molecule detaches electrons from negative ions with a known detachment rate.

For most spark discharges the production of these metastables has very little effect, because the lifetime of spark discharges is of the order of micro-seconds, which is of insufficient time for significant densities of metastables to develop. However, for a discharge of continuous pulses, as occurs in negative corona discharges, it is known that sustaining electric fields are markedly reduced from $\sim 25 \mathrm{kV} / \mathrm{cm}$ to $\sim 5 \mathrm{kV} / \mathrm{cm}$. Such significant changes can be explained by the development of significant densities of metastables in the discharge [3]. Figure 5 shows a predicted reduction in sustaining field from these metastables and Fig. 6 shows the high densities of metastables of the order of $10^{17} \mathrm{~cm}^{-3}$ that are required for such an effect.

The proposed theory of ball lightning relevant for the observations inside of houses and aircraft, is in three separate stages: (1) the accumulation of ions on 


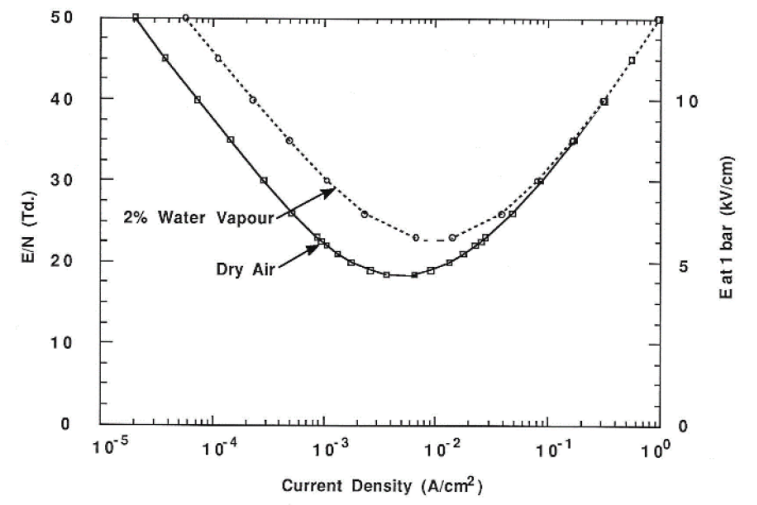

Figure 5. Metastables enable discharge operation at $5 \mathrm{kV} . \mathrm{cm}^{-1}$; from [3].

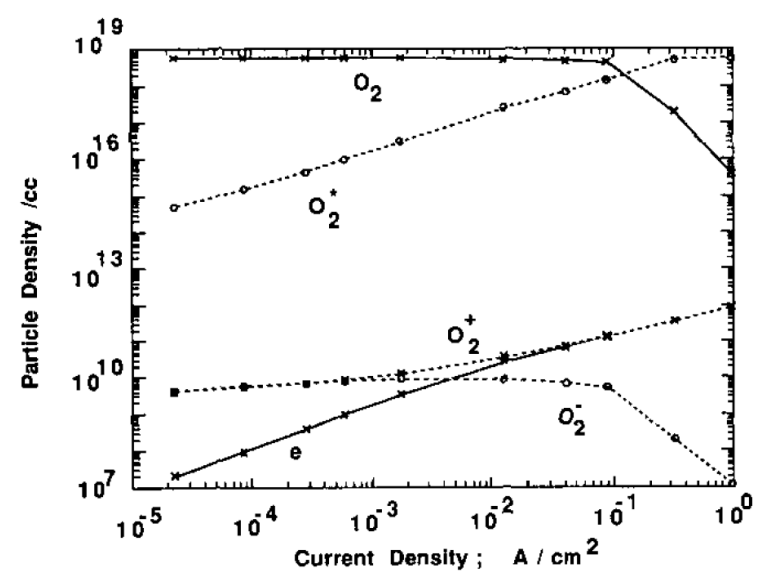

Figure 6. Equilibrium particle densities with metastables; from [3].

the external surface of a glass window, (2) initiation of pulsed discharges at the inside glass surface as in [4] and (3) the accumulation of high densities of metastables to produce ball lightning.

\subsection{Accumulation of ions on the glass window}

It is proposed that a stream of background atmospheric ions remaining from stalled stepped leaders of lightning are driven by electric fields to accumulate on the insulating surface of the outside of a glass window. Such calculated densities of the order of $10^{10} \mathrm{~cm}^{-3}$ are shown in Fig. 7a. Their effect is to double the background field on the other side of the glass, as shown in Fig. 7b. This mechanism was used to describe the initiation of discharges in the paper of [4].

\subsection{Discharge pulses at inside glass surface}

Fields greater than the breakdown electric field of $25 \mathrm{kV} / \mathrm{cm}$ are sufficient to initiate a discharge. Such discharges at low voltages are generally pulsed, known as Trichel pulses for negative potentials and a Hermstein discharge for positive potentials. Each discharge pulse will produce metastable molecules. Fig. 8 shows calculated electron density and thus
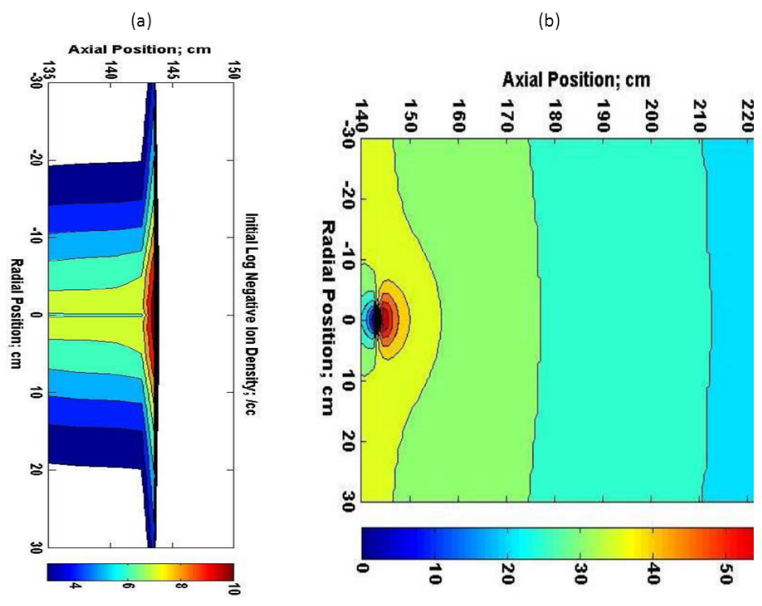

Figure 7. Calculated charge accumulation on outside of window at $143 \mathrm{~cm}$ shown at (a) and the field intensification inside of the window, shown at (b) in $k V . \mathrm{cm}^{-1}$.

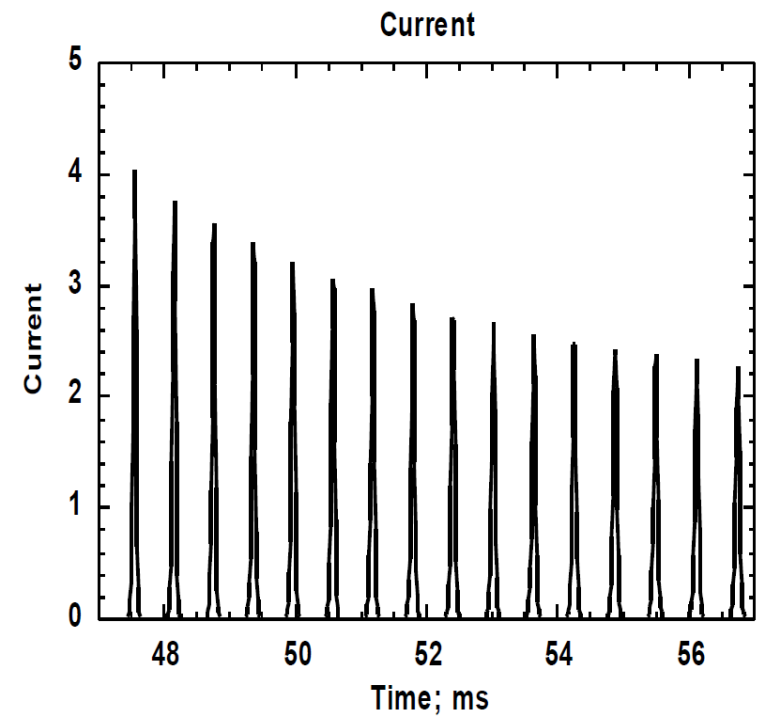

Figure 8. Calculated current pulses for Fig. 7 discharge.

current pulses in arbitrary units resulting from the dispersion of positive charges at the negative glass after each pulse.

\subsection{Accumulation of large metastable densities}

Metastables oxygen molecules will accumulate from each discharge pulse. From the discharge frequencies of Fig. 8, there would be $\sim 10,000$ pulses in 10 seconds. Calculations show, if metastable densities reach the order of $10^{17} \mathrm{~cm}^{-3}$, consistent with the steady state calculations of Fig. 6, electron densities from the detachment of negative ions by the metastables become of the same order or greater than the ion densities. These large electron densities then produce a completely changed distribution of the electric field, with the electric field within the ball becoming almost zero. Radiation will be emitted from the ball with a 


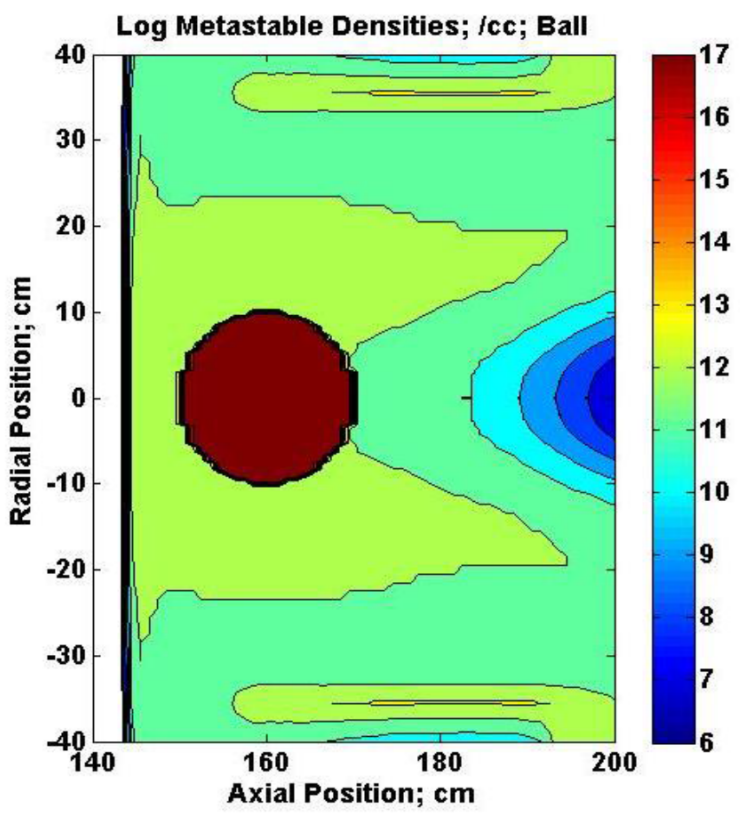

Figure 9. Calculated metastable density after insertion into the calculations of a ball of metastables of density $10^{17} \mathrm{~cm}^{-3}$.

lifetime dependent on the detachment coefficient of the metastables and the negative ion density.

Figures 9, 10 and 11 show calculated densities of metastables, electrons and negative ions after insertion into the calculations of a ball of metastables of density $10^{17} \mathrm{~cm}^{-3}$. The ball is assumed to be spherical due to the influence of diffusion of the metastables. The electron density then rises to $\sim 10^{10} \mathrm{~cm}^{-3}$, as shown in Figure 10, because of detachment of the negative ions by the metastables. The negative ion densities show a deficiency because of this detachment by the metastables, as shown in Fig. 11.

Figure 12 shows a remarkable reduction of the electric field to near zero within the ball and its surroundings, because of the high electrical conductivity of the electrons that are produced when the metastable density attains the density of the ions.

\section{Discharge plasmas to cure cancer?}

Traditional treatments of cancer in radiation therapy involve the bombardment of the cancer tumour with high energy particles. These high energy particles destroy the tumour cells which are impacted and also any healthy tissue that is also impacted. Recently evidence has accumulated that the impact of cancer tumours by discharges in air cause a reduction of tumour size. Not only is just the region directly impact by the plasma affected, but the whole tumour is reduced and there is even a suggestion of secondary tumours being influenced. Experimental efforts are being made to manufacture plasma sources that are safe for medical use. In particular oxygen metastables are being considered as possibly being the dominant

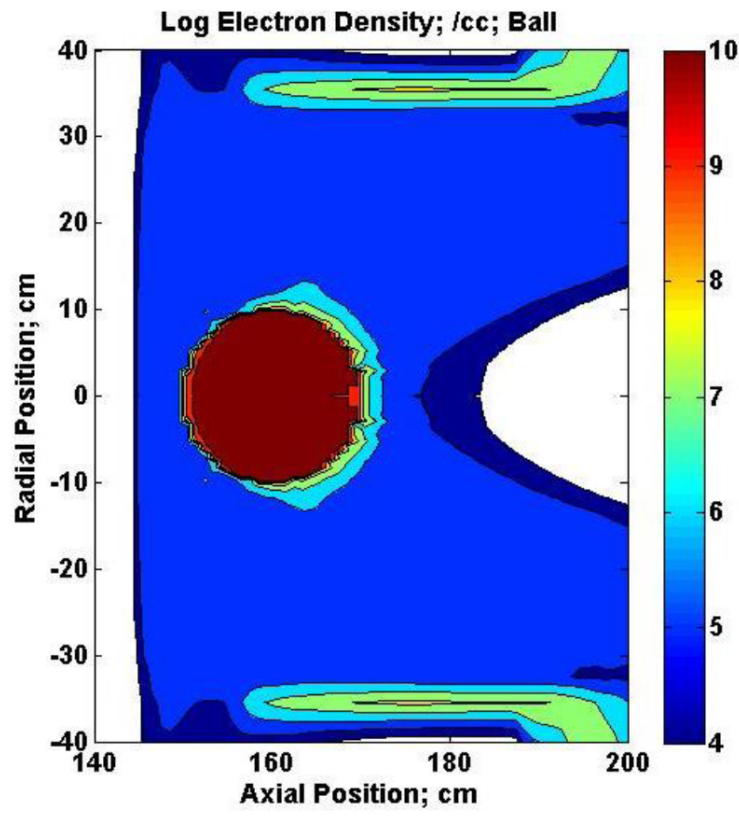

Figure 10. Calculated electron density after insertion into the calculations of a ball of metastables of density $10^{17} \mathrm{~cm}^{-3}$.

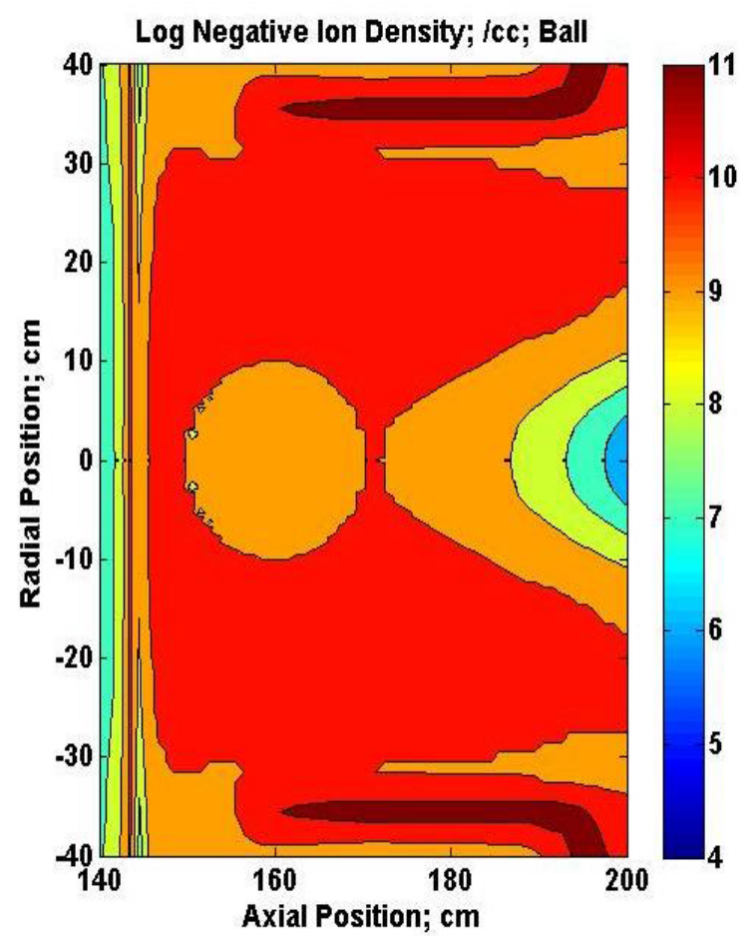

Figure 11. Calculated density of negative ions after insertion into the calculations of a ball of metastables of density $10^{17} \mathrm{~cm}^{-3}$.

active component. Efforts are being made to produce such plasmas that are not an electrical safety hazard and also produce significant concentrations of the oxygen metastables [5]. 


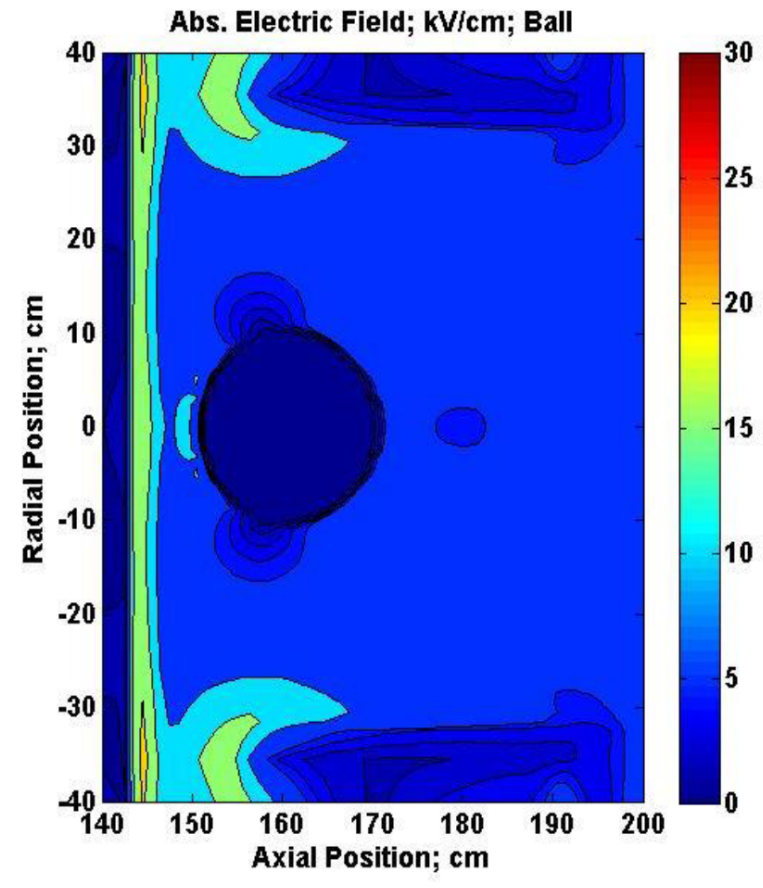

Figure 12. Electric field within the ball and its surroundings.

\section{Acknowledgements}

The author is grateful for comments given by Dr. A. B. Murphy of CSIRO throughout the course of this work.

\section{References}

[1] J. J. Lowke. Progress in arc theory, Proceedings of Sixth Int. Conf. on Switching Arc Phenomena, Lodz, Poland, Sept 12-15, 1989. In Sbornik, Bulletin Tech University Brno, pages 11-30, 1992.

[2] J. J. Lowke. Calculations of circuit interruption after current zero: Predictions of electron densities rather than temperatures. In Proc. 22nd Inter. Conf. on Gas Discharges and their Applications, volume 2, pages 347-350, 2018.

[3] J. J. Lowke. Theory of electrical breakdown in air the role of metastable oxygen molecules. J. Phys. D: Appl. Phys., 25(2):202-210, 1992. doi:10.1088/0022-3727/25/2/012/meta.

[4] J. J. Lowke, D. Smith, K. E. Nelson, C. R. W., and A. B. Murphy. Birth of ball lightning. J. Geophys. Res., 117:D19107, 2012. doi:10.1029/2012JD017921.

[5] J. S. Sousa, G. Bauville, and V. Puech. Arrays of microplasmas for the controlled production of tunable high fluxes of reactive oxygen species at atmospheric pressure. Plasma Sources Sci. Technol., 22:035012, 2013. doi:10.1088/0963-0252/22/3/035012. 